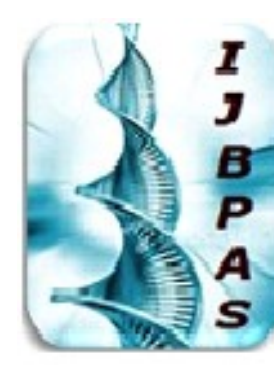

International Journal of Bhology, Pharmacy and Allied Sciences (IJBPAS) 'A B Bridge Betusen Cabratory and QRender'

www.ibpas.com

\title{
REGULATORY INFORMATION MANAGEMENT SYSTEM - A RAY OF HOPE IN DIGITAL PHARMA
}

\author{
NARAYANA CHARYULU $\mathbf{R}^{{ }^{*}}$, SWATHI ${ }^{1}$, SANDEEP DS ${ }^{1}$ AND MANOHAR $\mathbf{M}^{\mathbf{1}}$ \\ 1-Nitte (Deemed to be University), NGSM Institute of Pharmaceutical Sciences, Department of \\ Pharmaceutical Regulatory Affairs and Pharmaceutics, Paneer, Mangalore, Karnataka, India. \\ *Corresponding Author: RN Charyulu: E Mail: narayana@nitte.edu.in; Ph.: +919448164750 \\ Received $18^{\text {th }}$ March 2021; Revised $19^{\text {th }}$ April 2021; Accepted $8^{\text {th }}$ June 2021; Available online $1^{\text {st }}$ Feb. 2022 \\ https://doi.org/10.31032/IJBPAS/2022/11.2.5902
}

\begin{abstract}
Digitization influences all facets of our lives. Technology has been an essential part of the pharmaceutical industry in recent years. Every day, innovations and applications are introduced, resulting in a more sophisticated mode of communication. As a result, changes in consumer dynamics push the pharmaceutical industry to take the required measures to secure strong technological support that increases drug production and quality of life. A stable operating software system is updated regularly to minimize human error. Since the pharmaceutical industry operates in a highly organized environment, efficient data protection systems are necessary to prevent data integrity. The regulatory affairs professionals use semi-regulated systems, non-specialized software, organization-developed databases, and manual systems to support their daily operations. These ineffective methods handleseach product portfolio manually, resulting in unintentionalconcerns. Besides the strict regulatory environment, pharmaceutical companies must adopt a "Regulatory Information Management System" to handle the growing amount of data. The study below describes the prominent role of a regulatory information management system in the pharmaceutical industry to ensure data consistency and credibility as a requirement for implementing a risk management system. The main motto is to highlight the regulator's draught for the introduction and growth of "Regulatory Information Management"in pharmaceutical companies.
\end{abstract}

Keywords: Regulatory Management; Management Software; Modernization key; Attributes of software; Regulatory Compliance 


\section{INTRODUCTION}

Most recognized regulatory functional groups are critical for the dispatch of new drugs and explicitly allowing continued access to commercialized drug commodities.In recent years, regulatory compliance has received a great deal of attention from local and international governments. Compliance violations can lead to severe and sometimes catastrophic circumstances for the concerned organizations. Regulatory compliance has gained greater attention from the academic community in the last decade. Significant expenditures in organizations are associated with meeting regulatory compliance requirements. Compliance costs are one of the main issues that make companies reluctant to set up a compliance system [1].

The degree of compliance with regulations is a constantly increasing factor affecting the way all companies do business.IT departments face designing policies based on new standards while struggling to identify specific controls that can be successfully deployed within the organization. Several organizations are designing strategies for offering a "universal set of controls mapped into an industry-standard framework and then into the regulations". In the United States around the world, new regulations and legislation are applied everyday to companies. Such regulatory instruments affect various industries that require companies to implement certain safeguards and processes to ensure the privacy of customers and data security.Managing compliance with legislation is constantly tricky and expensive for organization around the world. While such efforts are often assisted by IT and information systems resources, there is proof that existing approaches are insufficient and do not fully address the organizational requirements. This discrepancy often resulted from a lack of coordination between the industry's needs and the emphasis of academic research efforts. Developing plans to navigate the inevitable regulatory changes that arise in the coming years from policy and global responses to the financial crisis will be high on corporate agendas [2]. Before, regulatory information was handled through regional and local regulatory bodies in many networks and files shares.

Consequently, getting a global overview of regulatory information had become a significant challenge for the industry. Regulatory affairs organization faces significant challenges in handling all of the information and communications relevant to 
global submissions and drug registration. In many cases, labour-intensive and manual processes seem to be time-consuming or costly, and error will be more. Outsourcing of partners must be regulated, and relevant regulatory filings must be revised and approved by health authorities. Regulatory adherence is an ever-growing factoraffecting how all organizations do business. Information security is one of the most criticalresponsibilitiesof corporations which can be achieved using practical tools such as RIMS [3].

In order to unify the submission process, ICH has made distinct progress in the form of CTD. CTD consists of five modules, out of which four are identical throughout all approving authorization, with module1 differeing by region. Besides, the US, Japan, Canada and several other countries are now approving pharmaceutical products via eCTD, which provides a data standard. Even though CTD and eCTD provide harmonization highly, significant variation still exists. The RIM system helps the organization simplify, streamline processes and develop best practices in Registration management, Regulatory intelligence. Tracking background information helps to see the bigger picture. Companies that have spent years buying a single system for each regulatory function consolidate them into a single RIM platform [4].

\section{THE CONCEPT BEHIND REGULATORY INFORMATION MANAGEMENT SYSTEM}

Regulatory Information Management Systems (RIMS) is a developing programming class that streamlines the submission of medicinal products for human use, including biopharmaceuticals, medical devices, pharmaceuticals, regulatory authorities, and finally, to gain approval to sell the said product in the desired market. Generally, RIMS is also termed regulatory information management software.

RIMS encompassesthe life cycle of the drug developmental process, from which companies effectively and efficiently create and manage information on a new or existing drug before, during, and after the registration. RIMS business key enables clients to access and deal with the information via a solitary user interface. IT approach to the RIM utilizes present systems to prevent a single point of failure and data transfer. The RIM is a pharmaceutical drug life cycle, including discovery, development, post-marketing surveillance, and critically complex. Combining the master data on goods and the documents associated with each step of the process is the core of process management. Mainly RIMS software system helps to 
manage Pharmaceutical product documents along with regulatory product information. It also assists in preparing Controlled submission of products and deals with submission of data to Regulatory authority in endorsed formats. A successful RIMS organizes the research required to obtain authorization for the sale of human medicinal products. Product submission may not seem critical at first glance, but the process of product submission is complex $[\mathbf{5 , 6}]$.

\section{ATTRIBUTES OF RIMS}

a) Maintain an audit trail following 21 CFR part 11 and also compliance with regulatory submission requirement.

b) Constructively manage the question raised by the regulatory authorities and track regulatory activities such as date of registration and expiry date.

c) Popular processes to create, compile, exchange, authorize, archive, review and maintain complete documents with minimum effort.

d) Effective dashboard-Status of different tasks like development, feedback, graphical overview of regulatory status, efficient event reminder.

e) Increase the accuracy and standard of pharmaceutical regulatoryknowledge [7].

\section{ADVANTAGES}

- Improved speed and authority of regulatory filing.

- Ideally structured regulatory process for pharmaceuticals.

- Effective software to replace manual monitoring.

- Eliminates dual data entry

- Access to all related product data and documents concerning the regulations.

- Helps in product registration monitoring and manage the due dates for registration.

\section{NEED OF RIMS}

RIMS providean easier and faster way to a market through more effective and efficient delivery and management of correspondence. It provides greater profitability for companies resulting from focusing on other tasks and simpler business process experiences with external service providers. This helps increase the global exposure of regulatory activities (Eg: what submissions were submitted in which countries, what promises were made, correspondence from the health authority, changes in legislation and many more).

RIMS enhances compliance of the product registration, helps to interact with the health authorities regularly, monitors product approvals globally, and regulates procurement throughout the product life 
cycle and monitors regulatory requests and promotional materials. It helps promote best practices in submission management and enhance coordination and process alignment of document authoring in countries where the applications have been accepted.RIMS help to receive updates inorder to monitor regulatory obligations and ensure they are fulfilled[8].RIMS help in the management of CMC - understanding the effect of manufacturing changes on regulatory filings and ensuring adequate approval before adoption by health authorities. As the anchor point for drug data and dossiers sent to regulators, today's RIMS system will play a much more pivotal role in regulatory approval process. RIMS as a central data repository improves regulatory approval processes by removing duplicate entries. RIMS identify the product information required by regulatory authorities and check the dossier setup [9].

RIMS provide consistent oversight life-cycle submission provides adequate support for the size and complexity of the business. RIM Software helps fulfil the need for the system's consolidation to create a single authoritative information source which helps in the globalization of main processes like product marking, handling and product registration, submission preparation and tracking. RIMS provide adaptability to evolving global regulations to provide the requisite scalability, convergence and single source of reality. The regulatory information collects and stores accurate product information based on industry-standard practices and enables approved users to track and manage the country and global registration data [10].

RIMINVESTMENT-generating significant returns on the introduction of an efficient RIM includes an initial investment. The technology-related costs solutions can be essential, mainly when a variety of point solutions are maintained by business [11].

TRANSFORMATIONAL VARIABLES FOR RIM-Harmonization, data visibility and collaboration are the main three forces for RIM transformation

a. Data visibility and reliability -A lack of corporate visibility is one of the effects of a fractured climate. Complete clarity is required for a regulatory team to provide proper guidance. Senior management must have instant access to the status of all post-marketing criteria and commitments. However, visibility is a significant challenge because knowledge is separated by geography and technology. According to the Gens study, basic questions like the status of a 
genuine commitment or a label shift at an affiliate office can take days or even weeks to reply. Another major problem in a decentralized landscape is data reliability, as local and regional teams are responsible for handling and preserving their data, which can lead to discrepancies at best and unreliable or obsolete information at worst, imparting pressure on regulatory teams to collect and check the most up-to-date data before accurately reacting, which can trigger submission delays. This burden is significantly reduced with a standard model for contributing data to global systems.

b. Collaboration-Many organizations are prioritizing improving link points between regulatory and other functional areas. Manufacturing changes, stock launches and label changes are all processes that depend on the ability to exchange and access accurate data, but they are all hindered by inefficiencies.Participants in the Gens study were asked to estimate the time it would take to provide reliable reporting for basic regulatory questions like the status of a label change in an affiliate or product renewal by product and region. c. Harmonization- Several independent RIM applications is a crucial factor in improving performance. Regulatory teams have historically operated in fragmented settings, leading to the widespread use of multiple independent applications to execute everyday tasks. Companies are transitioning from a fragmented RIM model in which structures differ by geography or product to a centralized RIM Model in which they use shared national systems and handle regulatory processes globally.Companies with a similar RIM model are 3.5 times more likely to recognize market advantages, according to Gens analysis, than those with disparate RIM environments [12].

\section{RIM MODERNISATION KEYS}

The following main levers are essential to modernize RIM to achieve improved regulatory, operational efficiency with reduced cost and time.

i. Riddance: As a critical element in achieving operational efficiency, process and IT landscape simplification has gained momentum. For most organizations, opportunities for development across regulatory information management systems due to metrics and lean methodologies are the 
current emphasis.Electronic Common Technical Document standardization enforcement and Fast Health Information Resources (FHIR) are some simplification tools.

ii. Digitalization: RIM has begun to embrace digital technology to process data and records in a more streamlined way. It is possible to track recordkeeping, share data and provide end to end traceability of regulatory events digitally.

iii. Automation: Automation is a true gamechanger for RIM conversion for manual, frequent, time-consuming and quality intensive processes.Various automation technologies, regulatory, operational effectiveness can be enhanced.

iv. Partnership:Implementing new working models, interfaces with internal and external regulatory stakeholders have become more significant. Besides the recent trend of outsourcing regulatory undertakings, it is challenging to handle partners concerned with information security issues. The significance of cloud-based collaboration systems with automated workflow has been stressed.

Today, the pharmaceutical industry faces anoperational challenge in product life cycle regulatory details. The difficulty is born from disconnected knowledge in software solutions that do not interact well in a disparate system. The complexity has compounded with the growth of emerging markets, multiple stakeholders, regional regulatory requirements and evolving enforcement needs. The inefficiency of the systems has been applied to method dependencies resulting in delayed submission, in turn, late market launch for drugs. It is no wonder that pharmaceutical companies are struggling to streamline the structure of the regulatory management system (RIM) [13].

Furthermore, the goal is to simplify and add versatility and maintain a holistic view of regulatory data from the discovery of the product to the launch. RIMS has the intellectual feature of evaluating the input material, which enables to obtain timely advice in the event of inconsistencies with the regulatory document requirements. It reduces the probability of errors to 30 percent. RIMS is continuously maintained and revised to reflect all current rules and regulations in the program's algorithms by leading experts on the regulatory practices. It also implements the possibility of integration with electronic data collection systems for patients and the research results. It also implements the possibility of integration with 
electronic data collection systems for patients and the research results. Automatic documents generation and control of each research subject are performed, leading to an increase in their conducting efficiency [14]. This software keepstrack of information submission and facilitates multi-user research and compliance with regulatory authorities requirements in various countries. A single database makes information secure for the long term; using a cloud service increases data storage capacity instead of local files on an individual employee's hard drive. This software can be combined with other systems and components to get pharmaceutical product description information.

\section{CONCLUSION}

The utilization of RIMS in combination with technical solutions to ensure data consistency is a prerequisite for implementing a risk management program and developing an efficient information generation process at the critical stages of the life cycle development of pharmaceuticals. This software is trending in the current scenario, and most pharmaceutical organizations are adapting this software to prevent data loss and save time. Though RIMS is developed based on regulatory requirement, it keeps baby steps in the digital Pharma world. It has its application and advantage to help the organization manage time, reduce the manual burden, and prevent duplicate or manual entries. RIMS need to be adopted by all the pharmaceutical industry in future so that data will be everlasting. As there isa need to improve the registration documents or implement pharmacovigilance, it is necessary to consider the quality and accuracy of such vital information relating to public health systems.

\section{CONFLICTS OF INTEREST}

The authors declare no conflict of interest.

\section{ACKNOWLEDGEMENTS}

The authors are grateful to the Nitte(Deemed to be University)NGSM Institute of Pharmaceutical Sciences, Department of Pharmaceutical Regulatory Affairs Deralakatte, Mangalore, for all the necessarysupport.

\section{REFERENCES}

[1] Babaei M; Beikzad J, Management Information System, Challenges and Solutions. European Online Journal of Natural and Social Sciences, 2(3), 2013, 374-381.

[2] Mattord H; Whitman ME, Regulatory compliance in Information Technology and Information Security. Americans Conference on Information Systems, 2007, 1-13. 
[3] Life Sciences Plus. Evolving Landscape of Regulatory Information Management in Pharma.[Internet] 2019 [Cited 2020 Feb 20].

https:/www.tcs.com/blogs/regulatoryinformation-management-in-pharma\#:

[4] Jacob D. LNS Research. What is Regulatory Information Management Software (RIMS)?[Internet]2019 [Cited 2020 Mar 02] Available from https://blog.lnsresearch.com/what-is$\underline{\text { regulatory-information-management- }}$ software

[5] Life sciences factsheet. Regulatory Information Management (RIM). [Internet] 2018 [Cited 2020 Feb 28]. https://www.cgi.com/sites/default/files/ $\underline{\text { 2018-08/cgi-us-life-sciences- }}$

regulatory-informationmanagement.pdf

[6] Regulatory information management system (RIMS): Keep your product data in control. [Internet]2019 [cited 2020 Mar 03].

https://www.nnit.dk/Life-

$\underline{\text { Sciences/Regulatory- }}$

Affairs/Sider/RIMS.

[7] Knowledge NET. Regulatory Information Management System (RIMS). [Internet] 2019 [Cited 2020 Mar 04] https://www.globalresearchonline.net/in strauthorspdf/IJPSRR_REFERENCE_F ORMAT.PDF

[8] Upton J. pharmexec. Regulatory information management: current and future states. [Internet] 2018 [Cited 2020 Mar 05]

http://www.pharmexec.com/regulatoryinformation-management-current-andfuture-states.

[9] Saha D,Regulatory Information Management: Novel Approaches for Health Care System. Bali Medical Journal, 2(3), 2013, 94-96.

[10] Abdullah N; Sadiq S; Indulska M. Advanced Information Systems Engineering. Emerging challenges in information systems research for regulatory compliance management. Pernici B editors, Heidelberg, Springer, 2010, 251-265.

[11] Deloitte. Growing Regulatory's Strategic Value. The Value of a Holistic Regulatory Information Management (RIM) capability. [Internet] 2018 [Cited 2020 Mar 07]. file://C:/Users/user/Downloads/regulatory-strategic-value\%20(7).pdf.

[12] Spaepen K, Attridge P. Uniting a fragmented RIM landscape: Best Practices for Managing Regulatory 
Processes. [Internet] 2017 [Cited 2020 Mar 12].

https://www.veeva.com/wp-

content/uploads/2017/10/Uniting-a-

fragmented-RIM-landscape.pdf

[13] Gronning N. Data management in a regulatory context. Frontiers in Medicine, 4, 2017, 1-5.

[14] Abdullah N, Sadiq S, Indulska M. Information systems research: Aligning to Industry Challenges in Management of Regulatory Compliance. Pacific Asia conference on information systems, 2010, 546-557. 